\title{
Influence of Oscillatory Impurity Potential and Concurrent Gasping of Impurity Spread on Excitation Profile of Doped Quantum Dots
}

\author{
Suvajit Pal ${ }^{1}$ and Manas Ghosh ${ }^{2}$ \\ ${ }^{1}$ Department of Chemistry, Hetampur Raj High School, Hetampur, Birbhum, West Bengal 731124, India \\ ${ }^{2}$ Department of Chemistry, Physical Chemistry Section, Visva-Bharati University, Santiniketan, Birbhum, West Bengal 731235, India \\ Correspondence should be addressed to Manas Ghosh; pcmg77@rediffmail.com
}

Received 12 December 2012; Accepted 19 January 2013

Academic Editor: Giancarlo Righini

Copyright (C) 2013 S. Pal and M. Ghosh. This is an open access article distributed under the Creative Commons Attribution License, which permits unrestricted use, distribution, and reproduction in any medium, provided the original work is properly cited.

Excitation in quantum dots is an important phenomenon. Realizing the importance we investigate the excitation behavior of a repulsive impurity-doped quantum dot induced by simultaneous oscillations of impurity potential and spatial stretch of impurity domain. The impurity potential has been assumed to have a Gaussian nature. The ratio of two oscillations $(\eta)$ has been exploited to understand the nature of excitation rate. Indeed it has been found that the said ratio could fabricate the excitation in a remarkable way. The present study also indicates attainment of stabilization in the excitation rate as soon as $\eta$ surpasses a threshold value regardless of the dopant location. However, within the stabilization zone we also observe maximization in the excitation rate at some typical location of dopant incorporation. The critical analysis of pertinent impurity parameters provides important perception about the physics behind the excitation process.

\section{Introduction}

Nowadays we frequently encounter a plethora of theoretical and experimental researches on impurity states in lowdimensional heterostructures [1]. The advent of so-called low-dimensional structures such as quantum dots (QD) has considerably accelerated the technology-driven demand of miniaturization of semiconductor devices. The quantized properties of quantum dots doped with impurity have generated new perspectives and subtleties in the field of applied physics. This happens because of the interplay between various confinement sources with impurity potentials [2]. Out of various kinds of investigations on impurity doping, control of optoelectronic properties emerges as the central theme [3-15]. Naturally, we find a vast literature comprising of good theoretical studies on impurity states [16-21]. Added to this, there are also some excellent experimental works which include the mechanism and control of dopant incorporation $[22,23]$.

Driven by the emergence of novel experimental and theoretical techniques along with the existing ones, the research on carrier dynamics in nanodevices has become a ubiquitous field. The internal transitions between impurity-induced states in a QD led to research on carrier dynamics $[8,24]$. These transitions depend on the spatial restriction imposed by the impurity. A minute survey of the above dynamical features directs us to explore excitation of electrons strongly confined in QD's. Analysis on this aspect deems importance since it offers us model systems for use in optoelectronic devices and as lasers. In connection with the technological applications, excitation in QD assumes importance in optical encoding, multiplexing, photovoltaic, and light-emitting devices. The phenomenon also plays some notable role in the eventual population transfer among the exciton states in QD $[25,26]$.

The aspects discussed above have urged us to investigate thoroughly excitation in doped quantum dots. As a result, of late, we have made some investigations on the excitation profiles of the doped quantum dots which were initiated by a time-dependent fluctuation of the impurity potential $\left(V_{0}\right)$ $[27,28]$. In these works we have studied the influence of interplay between the time-varying $V_{0}$ with impurity coordinate and spatial stretch of impurity on the excitation profile. 
The time variations of $V_{0}$ were taken to be periodic [27] and random [28] about its initial value. The origin of such a time variation of impurity potential was considered to be the environment inside the crystal lattice where the doped dot was nested. A deeper physical insight into our problem outlined so far reveals some deficiency. The deficiency results because of the deep interrelation between the spatial stretch of the dopant (denoted by symbol $\gamma$ in this paper) and the magnitude of dot-impurity interaction. A reduction in $V_{0}$ weakens the confines of electric $\left(\omega_{0}\right)$ and magnetic $\left(\omega_{c}\right)$ origins on the dopant whereas an increase in $V_{0}$ leads to a reverse consequence. Thus, a time-varying $V_{0}$ will surely be accompanied with a concomitant time-varying $\gamma$, and it appears to be too severe to consider $\gamma$ responding reluctantly to such a change of $V_{0}$. Of course, this time-dependent alteration in the spatial spread of dopant should not be arbitrary but must be guided by the fluctuation frequency of $V_{0}$. Thus, notwithstanding the tedious hike in the mathematical rigor we also incorporate the effect of time-dependent variation of $\gamma$ in our calculation. We conceive such a gasping impurity with a view to making the problem more realistic and rational. Recently we have done some works on this kind of time-dependent impurity spread in a different contexts $[29,30]$. Thus, in the present enquiry we explore the combined role of oscillatory impurity potential and impurity stretch on the said excitation profile. In view of this $V_{0}$ is allowed to oscillate with frequency $\left(v_{1}\right)$ enjoying the company of oscillatory $\gamma$ with frequency $\left(v_{2}=\right.$ $\eta v_{1}, \eta=$ integers and fractions). To be precise, in this paper we have monitored the ratio of the above two oscillation frequencies (termed as relative oscillation frequency, ROF) in connection with determining the time-average excitation rate for different dopant locations. In the present work the time dependence has been modeled by attaching a potential $V(t)$ to the dot Hamiltonian. A consequent followup of the dynamics of the doped dot in the time-dependent potential becomes the obvious next task to handle the problem. The observations elegantly reveal the combined effect of timedependent $V_{0}$ and $\gamma$ on the aforesaid excitation profile.

\section{Method}

We consider the energy eigenstates of an electron subject to a harmonic confinement potential $V(x, y)$ and a perpendicular magnetic field $B$, where $V(x, y)=(1 / 2) m^{*} \omega_{0}^{2}\left(x^{2}+y^{2}\right), \omega_{c}=$ $e B / m^{*} c$, and Landau gauge $(A=(B y, 0,0))$ has been used. $\omega_{0}, \omega_{c}$, and $A$ stand for harmonic confinement potential, cyclotron frequency (a measure of magnetic confinement offered by $B$ ), and vector potential, respectively. We have considered a magnetic field of mili Tesla (mT) order. The Hamiltonian in our problem reads

$$
\begin{aligned}
H_{0}^{\prime}= & -\frac{\hbar^{2}}{2 m^{*}}\left(\frac{\partial^{2}}{\partial x^{2}}+\frac{\partial^{2}}{\partial y^{2}}\right)+\frac{1}{2} m^{*} \omega_{0}^{2} x^{2} \\
& +\frac{1}{2} m^{*}\left(\omega_{0}^{2}+\omega_{c}^{2}\right) y^{2}-i \hbar \omega_{c} y \frac{\partial}{\partial x} .
\end{aligned}
$$

Define $\Omega^{2}=\omega_{0}^{2}+\omega_{c}^{2}$ as the effective frequency in the $y$ direction. $m^{*}$ is the effective electronic mass within the lattice of the material to be used. We have used $m^{*}=0.5 m_{0}$ and set $\hbar=e=m_{0}=a_{0}=1$. This $m^{*}$ value $\left(m^{*}=\right.$ 0.5 a.u.) closely resembles $\mathrm{Ge}$ quantum dots $\left(m^{*}=0.55\right.$ a.u.). The model Hamiltonian (cf. (1)) sensibly represents a 2 -d quantum dot with a single carrier electron [31,32]. The form of the confinement potential conforms to kind of lateral electrostatic confinement (parabolic) of the electrons in the $x-y$ plane $[9,10,16,33,34]$. In real QDs the electrons are confined in 3 dimensions; that is, the carriers do dynamically possess a quasi-zero-dimensional domain. The confinement length scales $R^{1}, R^{2}$, and $R^{3}$ can, in general, be different in three spatial directions, but usually $R^{3} \ll R^{1} \simeq R^{2}$. Whenever such QD's are modeled, $R^{3}$ is often taken to be strictly zero, and the confinement in the other two directions is described by a potential $V$ with $V(x) \rightarrow \infty$ for $|x| \rightarrow$ $\infty, x=\left(x^{1}, x^{2}\right) \in R^{2}$. A parabolic confinement potential, $V=(1 / 2) \omega|x|^{2}$, is often chosen as a realistic and the same time computationally convenient approximation. Assuming that the $z$ extension could be effectively considered zero, the electronic properties in these nanostructures have been successfully described using the model of 1-electron motion in 2-d harmonic oscillator potential in the presence of a magnetic field [31, 32].

Following earlier works on the effects of a repulsive scatterer in multicarrier dots in the presence of magnetic field $[35,36]$, here we have considered that the QD is doped with a repulsive Gaussian impurity. Now, as the impurity perturbation is attached to the Hamiltonian (cf. (1)), it transforms to

$$
H_{0}\left(x, y, \omega_{c}, \omega_{0}\right)=H_{0}^{\prime}\left(x, y, \omega_{c}, \omega_{0}\right)+V_{\mathrm{imp}}\left(x_{0}, y_{0}\right),
$$

where $V_{\text {imp }}\left(x_{0}, y_{0}\right)=V_{\text {imp }}(0)=V_{0} e^{-\gamma\left[\left(x-x_{0}\right)^{2}+\left(y-y_{0}\right)^{2}\right]}$ with $\gamma>0$ and $V_{0}>0$ for repulsive impurity and $\left(x_{0}, y_{0}\right)$ denotes the coordinate of the impurity center. $V_{0}$ is a measure of the strength of impurity potential and assumes a maximum value of $\sim 10^{-4}$ a.u. or $2.72 \mathrm{meV} . \gamma^{-1}$ determines the spatial stretch of the impurity potential. A large value of $\gamma$ indicates a highly quenched spatial extension of impurity potential whereas a small $\gamma$ accounts for spatially dispersed one. Thus, a change in $\gamma$ in turn causes a change in the extent of dot-impurity overlap that affects the excitation pattern noticeably [27-29]. The parameter $\gamma$ in the impurity potential is equivalent to $1 / d^{2}$, where $d$ is proportional to the width of the impurity potential $[35,36]$. The value of $\gamma$ is taken to be 0.001 a.u. which corresponds to an extension of the impurity domain up to $1.41 \mathrm{~nm}$. The presence of repulsive scatterer simulates dopant with excess electrons. The use of such Gaussian impurity potential is quite well known [37-39]. In view of the ongoing discussion the work of Gharaati and Khordad [40] merits mention. They introduced a new confinement potential for the spherical QD's called modified gaussian potential (MGP) and showed that this potential can predict the spectral energy and wave functions of a spherical quantum dot.

We write the trial wave function $\psi(x, y)$ as a superposition of the product of harmonic oscillator eigenfunctions $\phi_{n}(\alpha x)$ and $\phi_{m}(\beta y)$, respectively, as follows [29]:

$$
\psi(x, y)=\sum_{n, m} C_{n, m} \phi_{n}(\alpha x) \phi_{m}(\beta y),
$$


where $C_{n, m}$ are the variational parameters and $\alpha=$ $\sqrt{\left(m^{*} \omega_{0}\right) / \hbar}$ and $\beta=\sqrt{\left(m^{*} \Omega\right) / \hbar}$. In the linear variational calculation, we have used an appreciably large number of basis functions (cf. (3)) with $n, m=0-20$ for each of the directions $(x, y)$. This direct product basis spans a space of $(21 \times 21)$ dimension. It has been verified that the basis of such size scans the 2-d space effectively completely as long as monitoring the observables under investigation is concerned. A convergence test run by us with still greater number of basis functions confirmed our observation.

The general expression for the matrix elements of $H_{0}^{\prime}$ in the chosen basis is as follows:

$$
\begin{aligned}
&\left(H_{0}^{\prime}\right)_{n, m ; n^{\prime}, m^{\prime}} \\
&=\hbar\left\{\left(n^{\prime}+\frac{1}{2}\right) \omega_{0}+\left(m^{\prime}+\frac{1}{2}\right) \sqrt{\omega_{0}^{2}+\omega_{c}^{2}}\right\} \delta_{n, n^{\prime}} \delta_{m, m^{\prime}} \\
&-i \hbar \omega_{c} \frac{\alpha}{\beta}\left[\left\{\sqrt{\left.\frac{n^{\prime}}{2} \delta_{n^{\prime}-1, n}-\sqrt{\frac{n^{\prime}+1}{2}} \delta_{n^{\prime}+1, n}\right\}}\right.\right. \\
&\left.\cdot\left\{\sqrt{\frac{m^{\prime}+1}{2}} \delta_{m^{\prime}+1, m}+\sqrt{\frac{m^{\prime}}{2}} \delta_{m^{\prime}-1, m}\right\}\right] .
\end{aligned}
$$

And the matrix elements of $V_{\mathrm{imp}}$ are given by $[29,30]$

$$
\begin{aligned}
& \left(V_{\mathrm{imp}}\right)_{n, m ; n^{\prime}, m^{\prime}} \\
& \quad=V_{0} \cdot D_{1} \cdot D_{2} \cdot \sum_{k=0}^{\min \left(n, n^{\prime}\right)} \sum_{l=0}^{\min \left(m, m^{\prime}\right)} f\left(k, n, n^{\prime}\right) \cdot g\left(l, m, m^{\prime}\right),
\end{aligned}
$$

where

$$
\begin{aligned}
& f\left(k, n, n^{\prime}\right) \\
& \quad=2^{k} \cdot k ! \cdot{ }^{n} C_{k} \cdot{ }^{\prime} C_{k} \cdot\left(1-\alpha^{* 2}\right)^{\left(n+n^{\prime}\right) / 2-k} \cdot H_{n+n^{\prime}-2 k}\left(\alpha_{1} \rho_{1}\right), \\
& g\left(l, m, m^{\prime}\right) \\
& \quad=2^{l} \cdot l ! \cdot{ }^{m} C_{l} \cdot{ }^{m^{\prime}} C_{l} \cdot\left(1-\beta^{* 2}\right)^{\left(m+m^{\prime}\right) / 2-l} \cdot H_{m+m^{\prime}-2 l}\left(\beta_{1} \rho_{2}\right) .
\end{aligned}
$$

The other relevant quantities are $D_{1}=A \lambda_{1} \pi^{1 / 2} / \delta_{1}$, $D_{2}=B \lambda_{2} \pi^{1 / 2} / \delta_{2}, A=\alpha /\left(2^{n+n^{\prime}} n ! n^{\prime} ! \pi\right)^{1 / 2}, B=$ $\beta /\left(2^{m+m^{\prime}} m ! m^{\prime} ! \pi\right)^{1 / 2}, \delta_{1}^{2}=\alpha^{2}+\gamma, \delta_{2}^{2}=\beta^{2}+\gamma, \lambda_{1}=$ $\exp \left[-\gamma x_{0}^{2}\left(\delta_{1}^{2}-\gamma\right) / \delta_{1}^{2}\right]$, and $\lambda_{2}=\exp \left[-\gamma y_{0}^{2}\left(\delta_{2}^{2}-\gamma\right) / \delta_{2}^{2}\right], \rho_{1}=$ $\gamma x_{0} / \delta_{1}, \rho_{2}=\gamma y_{0} / \delta_{2}, \alpha^{*}=\alpha / \delta_{1}, \beta^{*}=\beta / \delta_{2}$. And $H_{n}(x)$ stands for the Hermite polynomials of $n$th order. The $p$ th eigenstate of the system in this representation can be written as

$$
\psi_{p}(x, y)=\sum_{i j} C_{i j, p}\left\{\phi_{i}(\alpha x) \phi_{j}(\beta y)\right\},
$$

where $i$ and $j$ are the appropriate quantum numbers, respectively, and $(i j)$ are composite indices specifying the direct product basis.
We can now introduce the time dependence into the impurity potential and impurity domain so that $V_{0} \rightarrow V_{0}(t)$ and $\gamma_{0} \rightarrow \gamma(t)$ [29]. Now the time-dependent Hamiltonian reads

$$
H(t)=\left[H_{0}-V_{\text {imp }}(0)\right]+V_{1}(t)
$$

where

$$
V_{1}(t)=V_{0}(t) e^{-\gamma(t)\left[\left(x-x_{0}\right)^{2}+\left(y-y_{0}\right)^{2}\right]} .
$$

The matrix element involving any two arbitrary eigenstates $p$ and $q$ of $H_{0}$ due to $V_{1}(t)$ reads (Details of derivation and various relevant quantities are given in references $[29,30])$ :

$$
\begin{aligned}
V_{p, q}^{\mathrm{imp}}(t)= & \left\langle\psi_{p}(x, y)\left|V_{1}(t)\right| \psi_{q}(x, y)\right\rangle \\
= & \sum_{n m} \sum_{n^{\prime} m^{\prime}} C_{n m, p}^{*} C_{n^{\prime} m^{\prime}, q}\left\langle\phi_{n}(\alpha x) \phi_{m}(\beta y)\left|V_{1}(t)\right|\right. \\
& \left.\quad \times \phi_{n^{\prime}}(\alpha x) \phi_{m^{\prime}}(\beta y)\right\rangle \\
= & V_{0}(t) \sum_{j=1}^{16} V_{p, q}^{j}(t) .
\end{aligned}
$$

We need to introduce now the explicit forms of time dependencies of $V_{0}$ and $\gamma$. The impurity potential strength $V_{0}$ is varied periodically with frequency $v_{1}$ as

$$
V_{0}=V_{0} \cos \left(v_{1} t\right) \text {. }
$$

Now, we consider the periodic fluctuation of $\gamma$ (viz. $\gamma(t)=$ $\left.\gamma_{0} \cos \left(\nu_{2} t\right)\right)$ as a result of fluctuation in $V_{0}$. The oscillation frequency of $\gamma$ (viz. $v_{2}$ ) has been considered to be multiples and submultiples of $\nu_{1}$ and can be represented as $v_{2}=\eta v_{1}$, $\eta=$ integers and fractions). The quantity $\eta$ could be termed as the relative oscillation frequency (ROF). Thus, the quantity $\eta$ actually links the two oscillations and eventually turns out to be a key factor in controlling the excitation rate.

Under the perturbation, the evolving wave function is described by a linear combination of the eigenstates of $H_{0}$ which is diagonal in the $\{\psi\}$ basis:

$$
\psi(x, y, t)=\sum_{q} a_{q}(t) \psi_{q}
$$

and we have to solve the TDSE:

$$
\begin{aligned}
& i \hbar \frac{\partial \psi}{\partial t}=H \psi \quad \text { or equivalently } \\
& i \hbar \dot{a}_{q}(t)=H a_{q}(t),
\end{aligned}
$$

to determine the time-dependent superposition coefficients with the initial conditions that $a_{p}(0)=1, a_{q}(0)=0$, for all $q \neq p$, where $p$ may be the ground or any other excited states of $H_{0}$. The time-dependent Schrödinger equation in the direct product basis (cf. (13)) has been integrated by the 6th-order Runge-Kutta-Fehlberg method with a time step size $\Delta t=0.01$ a.u., and the numerical stability of the 
integrator has been checked. The quantity $P_{k}(t)=\left|a_{k}(t)\right|^{2}$ indicates the population of $k$ th state of $H_{0}$ at time $t$. There occurs a continuous growth and decay in the ground state population $\left[P_{0}(t)\right]$ during the time evolution. Naturally the quantity $Q(t)=1-P_{0}(t)$ serves as a measure of excitation. In consequence, the quantity $R_{\mathrm{ex}}(t)=d \mathrm{Q} / d t$ serves as the timedependent rate of excitation. We have calculated the timeaverage rate of excitation $\left[\left\langle R_{\mathrm{ex}}\right\rangle=(1 / T) \int_{0}^{T} R_{\mathrm{ex}}(t) d t\right]$ with $T$ being the total time of dynamic evolution $(\sim 100 \mathrm{ps})$ as a function of ROF $(\eta)$ for different dopant locations $\left(r_{0}\right)$.

\section{Results and Discussion}

At the very outset of the discussion it needs to be realized that the excitation rate is controlled by the interplay between several factors of different characteristics. As the dopant is introduced at a greater distance from the dot confinement center $(0,0)$, the confines of electric $\left(\omega_{0}\right)$ and magnetic $\left(\omega_{c}\right)$ origins naturally become weak and favor excitation. On the other hand the said shift decreases the extent of repulsive interaction between the dot and the impurity, and excitation is unfavored. For a better visualization we have plotted the matrix elements $\left\langle\psi_{0}\left|\widehat{V}_{\text {imp }}\right| \psi_{0}\right\rangle$ as a function of radial position of impurity $\left(r_{0}\right)$ (Figure 1 ) to highlight the overlap of the impurity potential with the ground state wave function $\left|\psi_{0}\right\rangle$. The plot reveals that as the dopant is introduced away from the dot, their overlap decreases. However, at large $r_{0}$ the overlap settles to an otherwise steady value. The spatial stretch of impurity $(\gamma)$ has also a role to play in this context. An increase in $\gamma$ reduces the spatial stretch of the impurity potential which in turn reduces the extent of overlap between the dot and the impurity. Such a decrease in the said overlap has a two-pronged role, and primarily it reduces the dotimpurity interaction and consequently the strength of dot confinement thereby hindering and promoting the excitation at the same time. In this context the work of Xie merits mention which showed that absorptions in a spherical QD containing Gaussian impurity depend on electron-impurity overlap [41]. Thus, the excitation process is often found to be guided by factors of opposite natures arising out of the variation of several impurity parameters.

Let us now have a close look at the plot that delineates the time-average excitation rate $\left(\left\langle R_{\mathrm{ex}}\right\rangle\right)$ as a function of ROF $(\eta)$ for three different dopant locations, namely, on-center $\left(r_{0}=0.0\right.$ a.u. $)$, near off-center $\left(r_{0}=28.28\right.$ a.u. $)$, and far offcenter $\left(r_{0}=70.71\right.$ a.u.) (Figure 2$)$. It is evident from the figure that at all dopant locations the excitation rate culminates in a saturation at high $\eta$ values indicating a compromise between several factors that modulate the excitation. The diversities in the nature of excitation profiles are most prominent in low and medium $\eta$ values.

For an on-center dopant there is a considerable extent of dot-impurity overlap so that the role of $\gamma$ is undoubtedly important. Although at small and medium $\eta$ values the oscillations of $V_{0}$ are more pronounced than the oscillations of $\gamma$, it would be unwise to ignore $\gamma$ oscillation because of the close proximity of dot confinement center and the impurity coordinate. From the plot it comes out that at this dopant

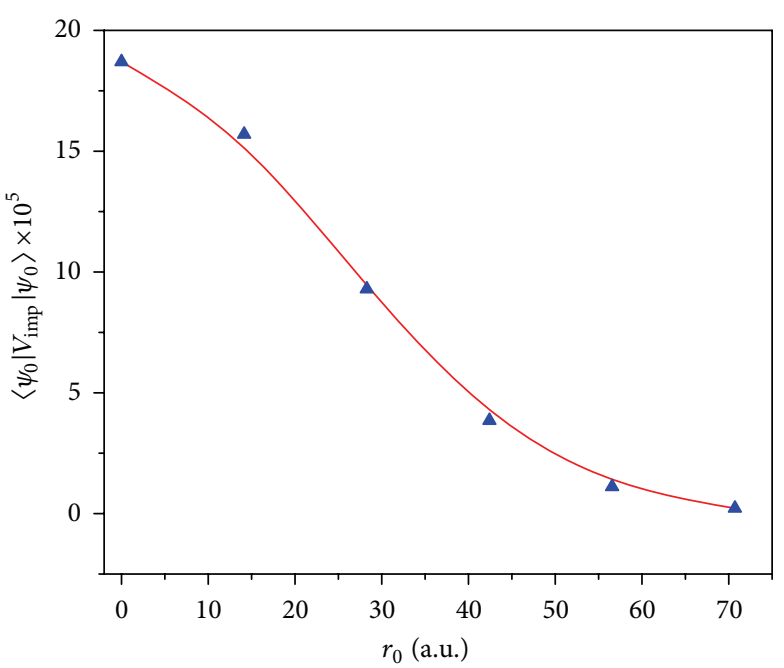

FIGURE 1: Plot of $\left\langle\psi_{0}\left|\widehat{V}_{\text {imp }}\right| \psi_{0}\right\rangle$ against $r_{0}$.

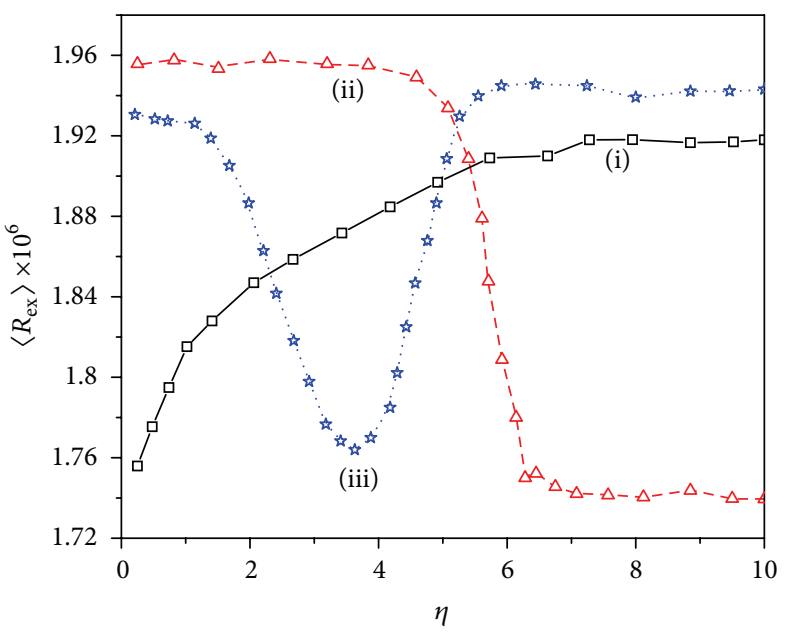

FIgURE 2: Plot of $\left\langle R_{\text {ex }}\right\rangle$ versus $\eta$ for different dopant locations $\left(r_{0}\right)$ with $\gamma_{0}=0.001$ a.u. and $V_{0}=1.0 \times 10^{-6}$ a.u. for (i) $r_{0}=0.0$ a.u., (ii) $r_{0}=28.28$ a.u., and (iii) $r_{0}=70.71$ a.u.

location, within small to medium $\eta$ domain, the combined oscillation of $V_{0}$ and $\gamma$ promotes excitation with increase in $\eta$ until there is a saturation. It comes out that by and large the $\gamma$ oscillation plays the governing role towards a steadfast enhancement of the excitation rate with increase in $\eta$. A gradual increase in $\eta$ in turn enhances $\gamma$ oscillation that ultimately causes steady increase in the excitation rate. Thus, as we realize from our earlier discussions that the role of $\gamma$ oscillation is not straightforward towards excitation, we perceive that, over and above, the factors that encourage excitation outweigh the opposite factors at these $\eta$ values. At large $\eta$ the $\gamma$ oscillation becomes predominant and invites some semblance of balance between the said factors leading to a saturation.

At near off-center dopant location the dot-impurity overlap gets somewhat reduced. Interestingly, at this dopant location we envisage saturation-like behavior of the excitation 


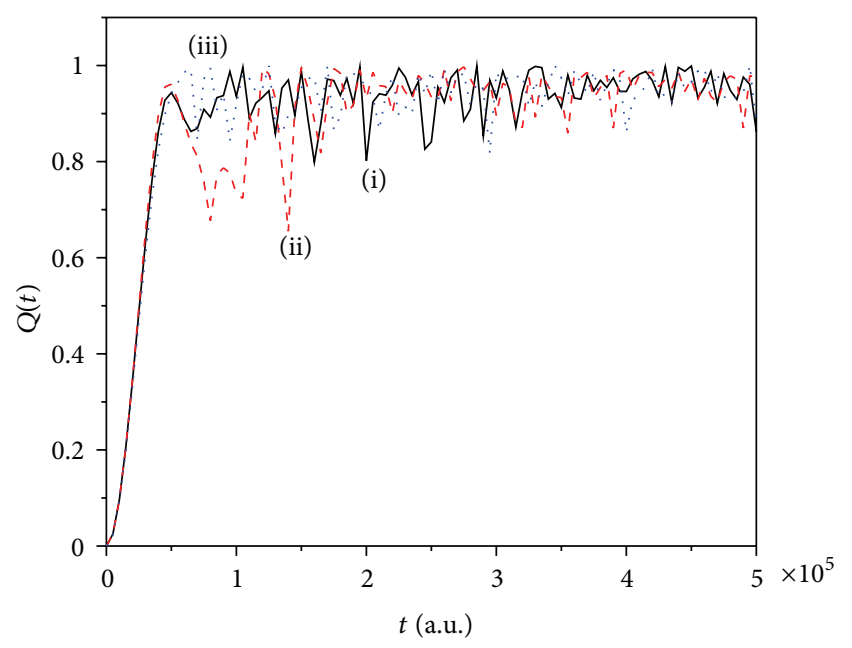

Figure 3: Plot of $Q(t)$ versus $t$ at $\eta=7.0$ for different dopant locations $\left(r_{0}\right)$ with $\gamma_{0}=0.001$ a.u. and $V_{0}=1.0 \times 10^{-6}$ a.u. for (i) $r_{0}=0.0$ a.u., (ii) $r_{0}=28.28$ a.u., and (iii) $r_{0}=70.71$ a.u.

rate at both low and high $\eta$ regimes. However, the magnitude of $\left\langle R_{\mathrm{ex}}\right\rangle$ differs noticeably in these two regimes. Whereas in low $\rightarrow$ medium $\eta$ domain $\left\langle R_{\mathrm{ex}}\right\rangle$ exhibits seemingly large values, in the neighborhood of $\eta \approx 5.0$ it undergoes a fall and beyond that $\left\langle R_{\mathrm{ex}}\right\rangle$ persists to register a rather low magnitude. The two domains where we observe such a saturation-like behavior are categorically distinguished from each other by simply a twist of relative dominance of $V_{0}$ and $\gamma$ oscillations. The factors emerging out of these oscillations interplay delicately so as to give rise to a steady excitation rate. However, looking at the placement of the dopant one must acknowledge that, over the entire range of $\eta$, the influence of $\gamma$ is not so much important as it is in case of an on-center dopant. It appears that in the vicinity of $\eta \approx 5.0$ the factors (principally owing to oscillatory $V_{0}$ ) that resist excitation completely outshine the reverse factors resulting in a depletion of the excitation rate. The $\gamma$ oscillation, since it plays the second fiddle through and through, becomes unable to resist the depletion.

For a far off-center dopant the excitation rate profile is endowed with a minima in the vicinity of $\eta \approx 4.0$. At such a distance the dot-impurity overlap gets highly quenched so that $\gamma$ oscillation remains almost indifferent towards excitation. Even at the large $\eta$ regime, where one usually expects $\gamma$ oscillation to be significant, seems to have very little impact owing to such a depleted dot-impurity overlap. However, at such a distant dopant location, both dot-impurity repulsive interaction and the strengths of confining forces are inherently weak. Thus, at such far off-center location there are factors of contradictory nature that can influence excitation. In low and medium $\eta$ zone, an increase in $\eta$ simply causes a lull in the $V_{0}$ oscillation. Since the dot-impurity interaction is inherently weak, the excitation rate decreases in compliance with the slowly decreasing $V_{0}$ oscillation. As soon as $\eta$ exceeds a threshold value of 4.0 , a change in the nature of excitation pattern comes into picture. The very fact that the dot confinement forces are weak enough now begins to favor excitation superseding all the effects that may hinder the process. The

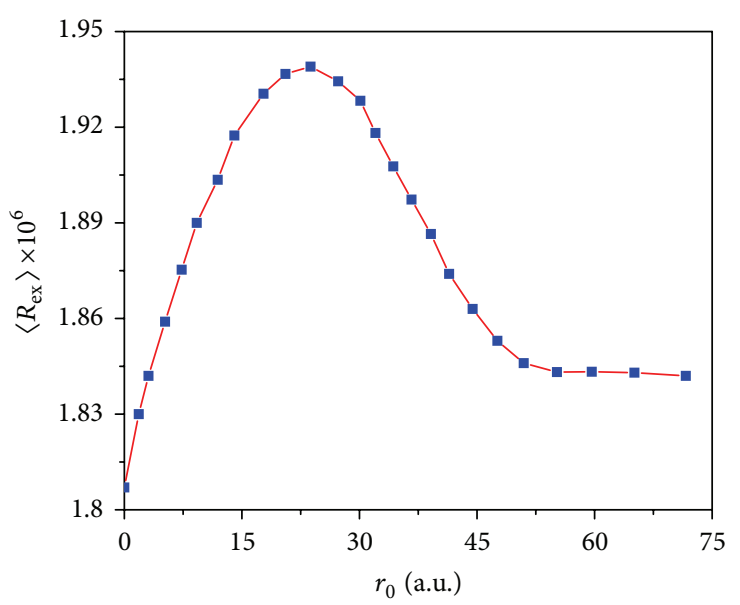

FIGURE 4: Plot of $\left\langle R_{\mathrm{ex}}\right\rangle$ versus $r_{0}$ at $\eta=7.0$ with $\gamma_{0}=0.001$ a.u. and $V_{0}=1.0 \times 10^{-6}$ a.u.

minima in the said profile thus turns out to be due to the changeover in the mutual dominance of factors that foment and impede excitation associated with a variation in ROF.

The important message hitherto received by us is that beyond $\eta \geq 6.0$ there occurs some kind of stabilization in $\left\langle R_{\mathrm{ex}}\right\rangle$ irrespective of dopant location. Thus, it goes without saying to conduct a more detailed inspection of this $\eta$ domain. Driven by this we have now monitored the excitation pattern $[Q(t)]$ as a function of time $(t)$ for above three dopant locations keeping $\eta$ fixed at 7.0 (Figure 3). From the plot it is evident that, at all dopant locations, excitation begins to increase sharply within a very short time once the perturbations are switched on. Then it exhibits some fluctuations as time advances and gradually gets damped on conclusion of the time evolution. Keeping $\eta$ fixed at this value also permits us to explore the exclusive dependence of excitation rate on dopant location when the said rate attains considerable stabilization.

In the present inspection the importance of dopant coordinate on excitation profile becomes perspicuous. In this connection the notable works of Baskoutas et al. [13], Karabulut and Baskoutas [14], and others [2,5] related to dopant located at off-center position are worth mentioning. Particularly, Karabulut, Baskoutas, and their coworkers studied the off-center impurities invoking an accurate numerical method potential morphing method (PMM). We, therefore, present now the solitary role of dopant location on the excitation pattern for a more comprehensive description of our investigation. Figure 4 exhibits the relevant $\left\langle R_{\mathrm{ex}}\right\rangle$ versus $r_{0}$ profile from which a distinct maximization in the excitation rate can be found in the neighborhood of a particular dopant coordinate $\left(r_{0} \approx 23\right.$ a.u. $)$. As we have pointed out earlier, the position-dependent maximization of the excitation rate occurs when the forces that resist excitation supersede the forces that have the reverse impact when the dopant is gradually shifted from on- to more and more off-center locations. Thus, in keeping with the works cited above, present investigation also divulges that a change in dopant location could bring about new hallmarks of excitation pattern. 


\section{Conclusions}

The excitation profile of repulsive impurity-doped quantum dots triggered by simultaneous oscillations of impurity potential, and impurity spread unveils noteworthy features. The ratio of two oscillations $(\eta)$ has been found to play governing role in influencing the excitation rate. Coupled with this, the dopant coordinate also modulates the excitation pattern to a considerable extent. We have found kind of stabilization in the excitation rate when $\eta$ exceeds some threshold value at all dopant locations. However, before that threshold value the excitation rates exhibit different trends dependend on the dopant coordinate. A critical analysis of $\eta$ domain when the excitation rate attains the steady behavior reveals the exclusive role played by the dopant coordinate towards excitation. The analysis evinced maximization in the excitation rate at some particular dopant coordinate. Whereas the maximization occurs due to a change in the relative preponderance of various factors that assist or resist excitation, the observed stabilization can be explained by arguing kind of compromise between the aforesaid factors. The results are thus quite interesting and expected to convey important insights in actual applications of quantum dot nanomaterials.

\section{Acknowledgments}

The authors S. Pal and M. Ghosh thank D. S. T-F. I. S. T (Govt. of India) and U. G. C.-S. A. P (Govt. of India) for partial financial support. Thanks are also due to Mr. Nirmal Kr Datta for his help.

\section{References}

[1] P. M. Koenraad and M. E. Flatté, "Single dopants in semiconductors," Nature Materials, vol. 10, no. 2, pp. 91-100, 2011.

[2] J. L. Movilla and J. Planelles, "Off-centering of hydrogenic impurities in quantum dots," Physical Review B, vol. 71, Article ID 075319, 7 pages, 2005.

[3] C. P. Poole Jr. and F. J. Owens, Introduction to Nanotechnology, John Wiley \& Sons, New York, NY, USA, 2003.

[4] M. J. Kelly, Low-Dimensional Semiconductors, Oxford University Press, Oxford, UK, 1995.

[5] W. Xie, "Nonlinear optical properties of an off-center donor in a quantum dot under applied magnetic field," Solid State Communications, vol. 151, no. 7, pp. 545-549, 2011.

[6] W. Xie, "Impurity effects on optical property of a spherical quantum dot in the presence of an electric field," Physica B, vol. 405, no. 16, pp. 3436-3440, 2010.

[7] R. Khordad, "Diamagnetic susceptibility of hydrogenic donor impurity in a $V$-groove $G a A s / G a_{1-x} A l_{x} A s$ quantum wire," European Physical Journal B, vol. 78, no. 3, pp. 399-403, 2010.

[8] C. A. Duque, N. Porras-Montenegro, Z. Barticevic, M. Pacheco, and L. E. Oliveira, "Effects of applied magnetic fields and hydrostatic pressure on the optical transitions in self-assembled InAs/GaAs quantum dots," Journal of Physics Condensed Matter, vol. 18, no. 6, pp. 1877-1884, 2006.

[9] Y. Yakar, B. Çakír, and A. Özmen, "Calculation of linear and nonlinear optical absorption coefficients of a spherical quantum dot with parabolic potential," Optics Communications, vol. 283, no. 9, pp. 1795-1800, 2010.
[10] A. J. Peter, "Polarizabilities of shallow donors in spherical quantum dots with parabolic confinement," Physics Letters A, vol. 355, no. 1, pp. 59-62, 2006.

[11] K. M. Kumar, A. J. Peter, and C. W. Lee, "Optical properties of a hydrogenic impurity in a confined $Z n_{1-x} C d_{x} S e / Z n S e$ spherical quantum dot," Superlattices and Microstructures, vol. 51, no. 1, pp. 184-193, 2012.

[12] B. Çakir, Y. Yakar, A. Özmen, M. Ö. Sezer, and M. Şahin, "Linear and nonlinear optical absorption coefficients and binding energy of a spherical quantum dot," Superlattices and Microstructures, vol. 47, no. 4, pp. 556-566, 2010.

[13] S. Baskoutas, E. Paspalakis, and A. F. Terzis, "Electronic structure and nonlinear optical rectification in a quantum dot: effects of impurities and external electric field," Journal of Physics Condensed Matter, vol. 19, no. 39, Article ID 395024, 9 pages, 2007.

[14] I. Karabulut and S. Baskoutas, "Second and third harmonic generation susceptibilities of spherical quantum dots: effects of impurities, electric field and size," Journal of Computational and Theoretical Nanoscience, vol. 6, no. 1, pp. 153-156, 2009.

[15] I. Karabulut and S. Baskoutas, "Linear and nonlinear optical absorption coefficients and refractive index changes in spherical quantum dots: effects of impurities, electric field, size, and optical intensity," Journal of Applied Physics, vol. 103, Article ID 073512, 5 pages, 2008.

[16] B. Gulveren, Ü. Atav, M. Sahin, and M. Tomak, "A parabolic quantum dot with $N$ electrons and an impurity," Physica E, vol. 30, pp. 143-149, 2005.

[17] E. Räsänen, J. Könemann, R. J. Haug, M. J. Puska, and R. M. Nieminen, "Impurity effects in quantum dots: toward quantitative modeling," Physical Review B, vol. 70, no. 11, Article ID 115308, 6 pages, 2004.

[18] M. Aichinger, S. A. Chin, E. Krotscheck, and E. Räsänen, "Effects of geometry and impurities on quantum rings in magnetic fields," Physical Review B, vol. 73, no. 19, Article ID 195310, 8 pages, 2006.

[19] S. Baskoutas and A. F. Terzis, "Binding energy of hydrogenic impurity states in an inverse parabolic quantum well under static external fields," European Physical Journal B, vol. 69, no. 2, pp. 237-244, 2009.

[20] F. J. Betancur, J. Sierra-Ortega, R. A. Escorcia, J. D. González, and I. D. Mikhailov, "Density of impurity states in doped spherical quantum dots," Physica E, vol. 23, no. 1-2, pp. 102-107, 2004.

[21] F. J. Betancur, I. D. Mikhailov, and L. E. Oliveira, "Shallow donor states in $G a A s-(G a, A l) A s$ quantum dots with different potential shapes," Journal of Physics D, vol. 31, p. 3391, 1998.

[22] S. V. Nistor, M. Stefan, L. C. Nistor, E. Goovaerts, and G. van Tendeloo, "Incorporation and localization of substitutional $\mathrm{Mn}^{2+}$ ions in cubic $\mathrm{ZnS}$ quantum dots," Physical Review B, vol. 81, no. 3, Article ID 035336, 6 pages, 2010.

[23] S. V. Nistor, L. C. Nistor, M. Stefan et al., "Synthesis and characterization of $\mathrm{Mn}^{2+}$ doped $\mathrm{ZnS}$ nanocrystals self-assembled in a tight mesoporous structure," Superlattices and Microstructures, vol. 46, no. 1-2, pp. 306-311, 2009.

[24] C. A. Duque, N. Porras-Montenegro, Z. Barticevic, M. Pacheco, and L. E. Oliveira, "Electron-hole transitions in self-assembled InAs/GaAs quantum dots: effects of applied magnetic fields and hydrostatic pressure," Microelectronics Journal, vol. 36, no. 3-6, pp. 231-233, 2005.

[25] E. Paspalakis and A. F. Terzis, "Controlled excitonic population transfer in a quantum dot system interacting with an electromagnetic field: local field effects," in Proceedings of the 5th 
International Conference on Microelectronics, Nanoelectronics and Optoelectronics, pp. 44-49, Prague, Czech Republic, 2006.

[26] A. Fountoulakis, A. F. Terzis, and E. Paspalakis, "Coherent single-electron transfer in coupled quantum dots," Journal of Applied Physics, vol. 106, no. 7, Article ID 074305, 8 pages, 2009.

[27] N. K. Datta and M. Ghosh, "Oscillatory impurity potential induced dynamics of doped quantum dots: analysis based on coupled influence of impurity coordinate and impurity influenced domain," Chemical Physics, vol. 372, no. 1-3, pp. 8288, 2010.

[28] N. K. Datta and M. Ghosh, "The randomly fluctuating impurity strength initiated excitation in doped quantum dots," Superlattices and Microstructures, vol. 51, no. 5, pp. 690-698, 2012.

[29] N. K. Datta, S. Pal, and M. Ghosh, "Influence of relative confinement oscillation and concomitant oscillatory impurity domain on excitation profile of doped quantum dots," Chemical Physics, vol. 400, pp. 44-50, 2012.

[30] N. K. Datta, S. Pal, and M. Ghosh, "Influence of impurity propagation and concomitant enhancement of impurity spread on excitation profile of doped quantum dots," Journal of Applied Physics, vol. 112, no. 1, Article ID 014324, 8 pages, 2012.

[31] L. Jacak, P. Hawrylak, and A. Wojos, Quantum Dots, Springer, Berlin, Germany, 1998.

[32] T. Chakraborty, Quantum Dots-A Survey of the Properties of Artificial Atoms, Elsevier, Amsterdam, The Netherlands, 1999.

[33] W. Xie and J. Gu, "Exciton bound to a neutral donor in parabolic quantum dots," Physics Letters A, vol. 312, no. 5-6, pp. 385-390, 2003.

[34] S. Baskoutas, A. F. Terzis, and E. Voutsinas, "Binding energy of donor states in a quantum dot with parabolic confinement," Journal of Computational and Theoretical Nanoscience, vol. 1, no. 3, pp. 317-321, 2004.

[35] V. Halonen, P. Hyvönen, P. Pietiläinen, and T. Chakraborty, "Effects of scattering centers on the energy spectrum of a quantum dot," Physical Review B, vol. 53, no. 11, pp. 6971-6974, 1996.

[36] V. Halonen, P. Pietiläinen, and T. Chakraborty, "Opticalabsorption spectra of quantum dots and rings with a repulsive scattering centre," Europhysics Letters, vol. 33, no. 5, pp. 377-382, 1996.

[37] J. Adamowski, A. Kwaśniowski, and B. Szafran, "LO-phononinduced screening of electron-electron interaction in $D^{-}$centres and quantum dots," Journal of Physics Condensed Matter, vol. 17, no. 28, pp. 4489-4500, 2005.

[38] S. Bednarek, B. Szafran, K. Lis, and J. Adamowski, "Modeling of electronic properties of electrostatic quantum dots," Physical Review B, vol. 68, no. 15, Article ID 155333, 9 pages, 2003.

[39] B. Szafran, S. Bednarek, and J. Adamowski, "Parity symmetry and energy spectrum of excitons in coupled self-assembled quantum dots," Physical Review B, vol. 64, no. 12, Article ID 125301, 10 pages, 2001.

[40] A. Gharaati and R. Khordad, "A new confinement potential in spherical quantum dots: modified gaussian potential," Superlattices and Microstructures, vol. 48, no. 3, pp. 276-287, 2010.

[41] W. Xie, "Excited state absorptions of an exciton bound to an ionized donor impurity in quantum dots," Optics Communications, vol. 284 , no. 24 , pp. $5730-5733,2011$. 

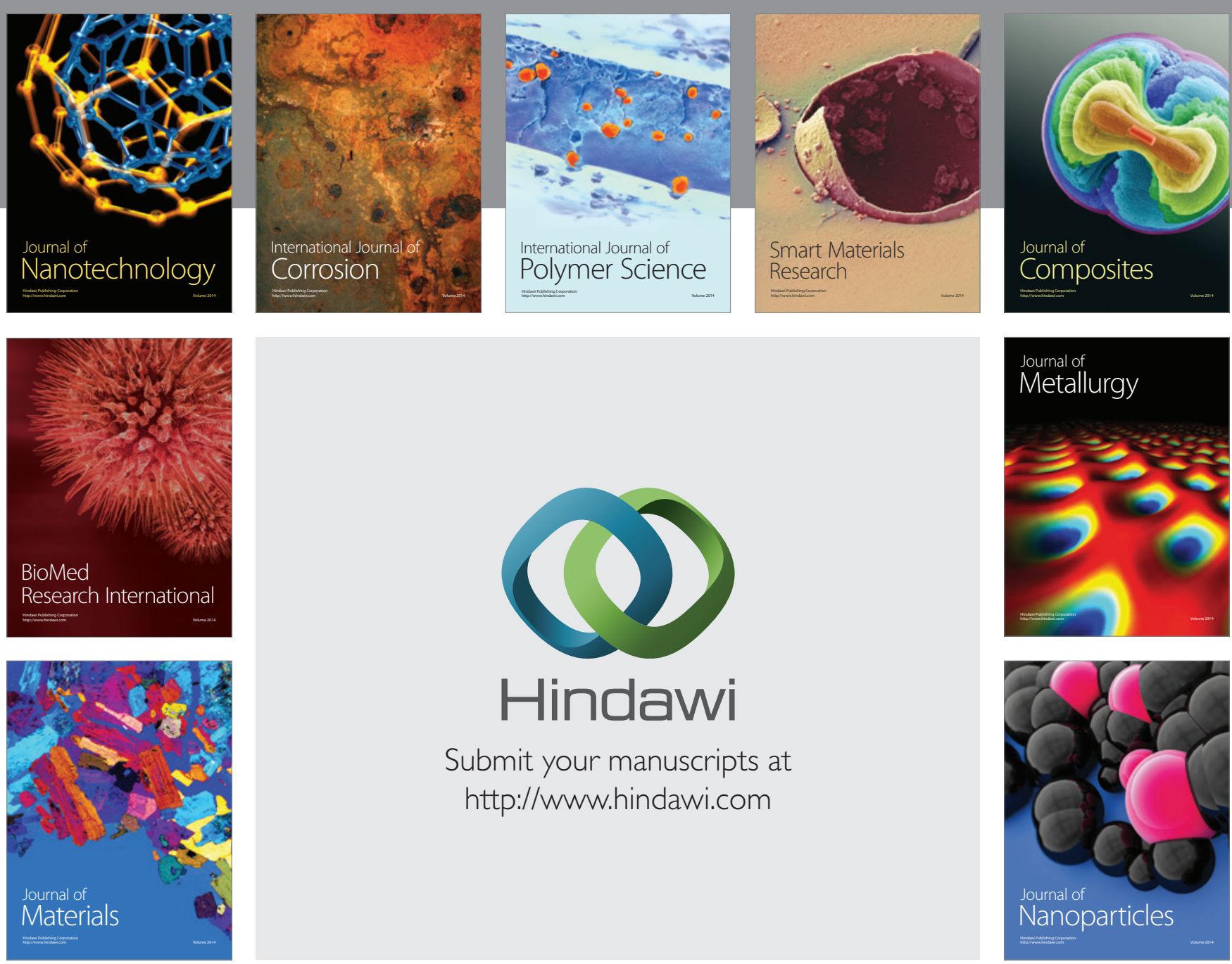

Submit your manuscripts at http://www.hindawi.com
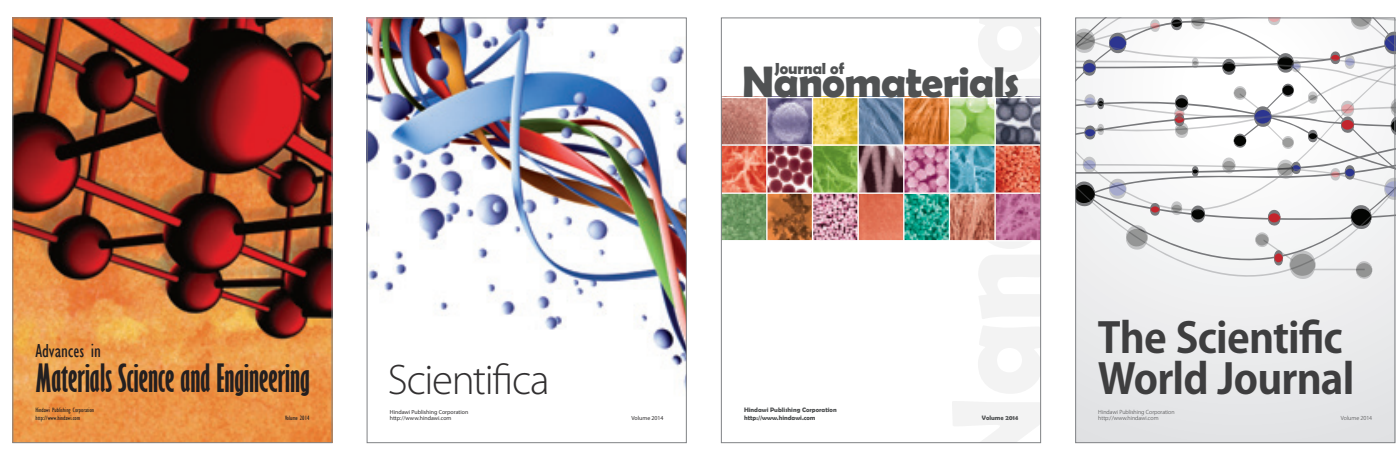

\section{The Scientific World Journal}
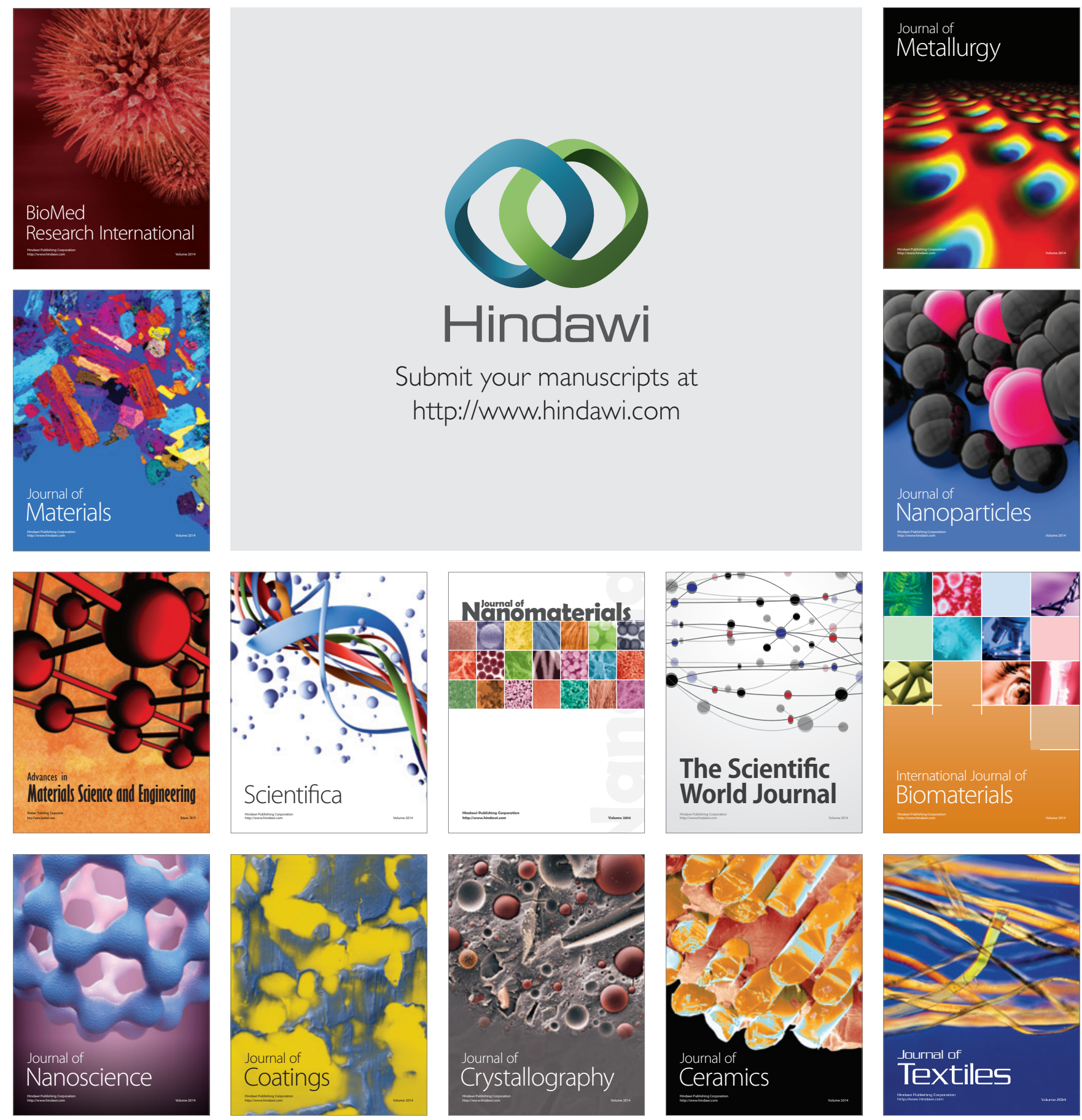